ICN-UNAM-97-03

July 11, 1997

\title{
Regge Behaviour from an Environmentally Friendly Renormalization Group
}

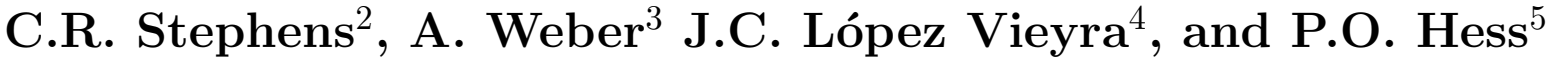 \\ Instituto de Ciencias Nucleares, U.N.A.M., \\ A. Postal 70-543, 04510 Mexico D.F., Mexico.
}

\begin{abstract}
The asymptotic behaviour of cubic field theories is investigated in the Regge limit using the techniques of environmentally friendly renormalization, environmentally friendly in the present context meaning asymmetric in its momentum dependence. In particular we consider the crossover between large and small energies at fixed momentum transfer for a model scalar theory of the type $\phi^{2} \psi$. The asymptotic forms of the crossover scaling functions are exhibited for all two particle scattering processes in this channel to one loop in a renormalization group improved perturbation theory.
\end{abstract}

\footnotetext{
${ }^{1}$ This work was supported by Conacyt grant 3298P-E9608.

${ }^{2} \mathrm{e}-$ mail: stephens@nuclecu.unam.mx

${ }^{3}$ Supported by fellowships of the DAAD and the Mexican Government; e-mail: axel@nuclecu.unam.mx

${ }^{4} \mathrm{e}$-mail: vieyra@nuclecu.unam.mx

${ }^{5} \mathrm{e}-\mathrm{mail}:$ hess@nuclecu.unam.mx
} 
One of the most active areas of interest in QCD both experimentally and theoretically is the limit $Q^{2} \gg \Lambda_{Q C D}^{2}, x \ll 1$ where $Q^{2}$ and $x$ are the Bjorken scaling variables. This corresponds to the diffractive, or Regge limit, where standard renormalization group (RG) improved perturbation theory breaks down. Various methods have been proposed and used to tackle this problem with varying degrees of success. One of the most common is the summation of leading logs [1] via an inspection of the perturbation expansion, a technique which has a long history (see for instance [2] and references therein). Besides an intrinsic degree of arbitrariness, summing sets of $\operatorname{logs}$ can be quite difficult combinatorially in many cases.

The RG has proved to be an extremely useful non-perturbative tool, especially in the context of non-abelian gauge theories where the ultraviolet fixed point can be accessed via very simple renormalization procedures such as minimal subtraction and has had great success when applied to deep-inelastic scattering. In the Regge limit however, the RG has been conspicuous by its absence, except for the beautiful application to Reggeonic field theory [3]. The principal reason is its association with ultraviolet divergences. In the Regge limit the breakdown of perturbation theory has nothing to do with the latter, however, terms such as $\ln s$ or $\ln t$ in the limits $s, t \rightarrow \infty$ do lead to divergences. These divergences in contradistinction to short distance behaviour are very asymmetric. The reason they appear can be traced to the nature of the effective degrees of freedom in the problem. For small $s$ and $t$ they are four dimensional, whereas in the Regge limit, as is well known, there is a "kinematic" dimensional reduction to two dimensions owing to the extreme anisotropy between the longitudinal and transverse sectors. Such crossovers between effective degrees of freedom of one type and another, qualitatively completely different, are ubiquitous in physics. Indeed the crossover between asymptotic freedom and confinement offers a perfect paradigm.

To describe systematically such crossovers using RG methods one requires a RG that can interpolate between different effective degrees of freedom as a function of "scale", where scale could mean temperature, momentum, size etc. Such an RG, that can be applied to a myriad of other crossover situations, has been developed under the name of "environmentally friendly" renormalization [4] in recognition of the fact that a crossover very often can be thought of as taking place due to the effect of some "environmental" parameter, such as temperature. Using these methods it has been possible, for instance, to access the dimensional crossover in finite temperature field theory [5] between an effective four dimensional theory at low temperatures to an effectively three dimensional theory near a second or weakly first order phase transition. The purpose of this letter is to present an environmentally friendly renormalization that can access the non-perturbative crossover to Regge behaviour in the context of a scalar cubic model theory. Applications to QCD will be considered in other publications.

First we will present the basic renormalization scheme we will use to treat the $t$-channel crossover between "small" $t \sim s$ and large $t \gg s$ for fixed $s$, where $s$ is a momentum variable in the physical region. The results we present can also be applied to the $s$-channel crossover beween small and large $s$. The theory we will consider is a simple "mesonic" cubic theory where we can ignore the effects of spin. We will consider an interaction of the form $\left(g_{B} / 2\right) \phi^{2} \psi$ where the $\phi$ and $\psi$ fields have bare masses $m_{B}$ and $M_{B}$ respectively. Results for the cases $\phi^{3}, \phi^{\dagger} \phi \psi$ and a Wick-Cutkosky type model $\sum_{i=1}^{2} \phi_{i}^{\dagger} \phi_{i} \psi$ will follow quite simply from the case 
treated.

The function of interest here will be the connected four point function $G^{i j k l}$ on shell. We use the superscript notation $i j k l$ to denote the external legs, i.e. $i, j, k, l$ can take the values $\phi$ or $\psi$. The last two indices refer to the incoming and the first two to the outgoing particles in the $s$ channel as shown in figure 1. To simplify matters we may remove the external legs to define

$$
\tilde{\Gamma}_{B}^{i j k l}\left(p_{1}, p_{2}, p_{3}, p_{4}\right)=\Gamma_{B}^{i i}\left(p_{1}\right) \Gamma_{B}^{j j}\left(p_{2}\right) \Gamma_{B}^{k k}\left(p_{3}\right) \Gamma_{B}^{l l}\left(p_{4}\right) G_{B}^{i j k l}\left(p_{1}, p_{2}, p_{3}, p_{4}\right) .
$$

The relation to fully one-particle irreducible vertex functions is via

$$
\begin{aligned}
\tilde{\Gamma}_{B}^{i j k l}(s, t, u)= & \Gamma_{B}^{i j k l}(s, t, u)+\Gamma_{B}^{i j m}(s) G_{B}^{m m}(s) \Gamma_{B}^{m k l}(s) \\
& +\Gamma_{B}^{i k m}(t) G_{B}^{m m}(t) \Gamma_{B}^{m j l}(t)+\Gamma_{B}^{i l m}(u) G_{B}^{m m}(u) \Gamma_{B}^{m j k}(u)
\end{aligned}
$$

for the external momenta on mass shell. One may further decompose the irreducible four point function in the following way (see below):

$$
\Gamma_{B}^{i j k l}(s, t, u)=A_{B, t}^{i j k l}(s, t)+A_{B, u}^{i j k l}(s, u)+A_{B, s}^{i j k l}(t, u) .
$$

Now we come to the question of renormalization of these functions. We will consider $d=4$. As far as ultraviolet divergences are concerned the only badly behaved diagrams are those that contain radiative corrections to the masses, however for $t \gg s$, at $n$-loop order, there exist diagrams which give corrections of $O\left((\ln t)^{n}\right)$. In the limit $t \rightarrow \infty$ we thus have new divergences which have nothing to do with the ultraviolet. As mentioned in the introduction they are a symptom of the fact that the correct effective degrees of freedom of this model for asymptotically large $t$ are completely different to those of the small $t$ region. This is due to the fact that the transverse degrees of freedom become strongly coupled leading to an effective "dimensional reduction", or perhaps better to say "factorization", of the loop graphs into the form $g(t) K(s)$ where $g(t)$, associated with the longitudinal direction is effectively two-dimensional, whilst $K(s)$, associated with the transverse dimensions, is $(d-2)$-dimensional. Indeed, the dimensional crossover shares several features in common with that of say $\lambda \phi^{4}$ theory on $S^{1} \times S^{1} \times R^{d-2}$, where the four point coupling is to one loop

$$
\Gamma_{B}^{(4)}=\lambda_{B}-\frac{3}{2} \frac{\lambda_{B}^{2}}{L^{2}} \sum_{n_{1}, n_{2}} \int \frac{d^{d-2} k}{\left(k^{2}+m^{2}+\frac{4 \pi^{2}\left(n_{1}^{2}+n_{2}^{2}\right)}{L^{2}}\right)^{2}} .
$$

In the limit $L m \ll 1$ one can neglect other than the zero eigenmodes and so one has a factorization into a $(d-2)$-dimensional loop integral and an $L$ dependent effective coupling constant $g_{B}=\lambda_{B} / L^{2}$. In this case for $L m \gg 1$ the effective degrees of freedom of the system are $d$-dimensional whilst for $L m \ll 1$ they are $(d-2)$-dimensional. The effective degrees of freedom being environment dependent, i.e. $L$ dependent, require an $L$ dependent renormalization that is capable of capturing both a $d$ and $(d-2)$-dimensional fixed point. Such a renormalization it should be emphasized is highly anisotropic in that the relevant counterterms, unlike for instance minimal subtraction type counterterms, are very sensitive to the asymmetry between the finite and infinite directions. In the case at hand the effective degrees of freedom are strongly $t$ dependent hence an asymmetric renormalization that 
can take into account the anisotropy between the longitudinal and transverse directions is required. Clearly any renormalization scheme symmetric in the momenta such as used for the running coupling in QCD will be totally inadequate.

The vertex correction in the $t$ channel (see eq. (2)) varies like $\left(g_{B}^{2} / t\right)^{n}(\ln t)^{2 n}$ in four dimensions at $n$ loop order. Thus there is no Sudakov suppression here and the bare vertex will give a good approximation. A similar argument holds true in the $u$ channel where $u \rightarrow-t$ asymptotically. In the $s$ channel the corrections do not go to zero, however, they are small in the sense that they are perturbatively controllable. For mass renormalization of the $\psi$ field one may remove the ultraviolet divergence via a mass shell renormalization, similarly for the mass divergence of the $\phi$ field. In the $t$ channel the resulting $n$-loop contribution to $G_{B}^{i i}$ is $\sim\left(g_{B}^{2} / t\right)^{n}\left((\ln t)^{n} / t\right)$. Thus the momentum dependent corrections after an ultraviolet subtraction go to zero as $t \rightarrow \infty$. The same holds true in the $u$ channel, whilst in the $s$ channel the corrrections are non-zero but once again are perturbatively controllable. The upshot of all this is that the crossover to dimensionally reduced behaviour in the large $t$ limit is controlled by ladder-type diagrams. This is interesting in that renormalization of the theory cannot now be achieved by a reparametrization of the original parameters of the theory, $g_{B}, m_{B}$ and $M_{B}$.

One concludes that a renormalization of the effective four point interaction itself is required. There is nothing particularly strange about this. One can easily convince oneself of its naturalness by considering the limit $M \rightarrow \infty, m \rightarrow 0$ after renormalizing the theory as a cubic theory. Physically one knows that an effective $d$-dimensional $\lambda \phi^{4}$ theory has to emerge. However, renormalization of $m_{B}$ and $g_{B}$ is not sufficient to remove the characteristic infrared divergences for $d \leq 4$. A subsequent renormalization of the four point vertex itself is required in order to arrive at the usual $\phi^{4}$ renormalization constants. This renormalization is associated only with the one-particle irreducible part, given at one loop by the "box" diagrams. Of course, this limit is completely opposite to the one we are considering here, i.e. the Regge limit. It simply illustrates that there exists another kinematical regime wherein one sees that renormalization of the parameters of the model is not sufficient to render the theory perturbatively tractable.

There are two ways we can proceed now, by renormalizing $\tilde{\Gamma}_{B}^{i j k l}$ directly or via a renormalization of $\Gamma_{B}^{i j k l}$. Here we will consider the renormalization at the level of the functions $A$ above in (3), or more precisely for the functions $B$ defined via

$$
\begin{gathered}
B_{B, t}^{i j k l}(s, t)=A_{B, t}^{i j k l}(s, t)+\Gamma_{B}^{i k m}(t) G_{B}^{m m}(t) \Gamma_{B}^{m j l}(t), \\
B_{B, u}^{i j k l}(s, u)=A_{B, u}^{i j k l}(s, u)+\Gamma_{B}^{i l m}(u) G_{B}^{m m}(u) \Gamma_{B}^{m j k}(u), \\
B_{B, s}^{i j k l}(t, u)=A_{B, s}^{i j k l}(t, u)+\Gamma_{B}^{i j m}(s) G_{B}^{m m}(s) \Gamma_{B}^{m k l}(s)
\end{gathered}
$$

A diagram contributing to $\Gamma_{B}$ is by definition associated with $A_{B, t}$ if it contains powers of $\ln t$ in the large $t$ limit, with $A_{B, u}$ if it contains powers of $\ln (-t)$, and with $A_{B, s}$ otherwise.

We now wish to define renormalized functions $B^{i j k l}$, restricting attention to $B_{t}^{i j k l}$ for the moment. Due to mixing of the effective cuartic interactions with different $i j k l$ a pure 
multiplicative renormalization is not sufficient, one must introduce a matrix renormalization via

$$
B_{t}^{i j k l}(s, t, g(\kappa), m(\kappa), M(\kappa), \kappa)=\sum_{m, n} Z_{b, t}^{i j m n}(\kappa) B_{B, t}^{m n k l}\left(s, t, g_{B}, m_{B}, M_{B}, \Lambda\right)
$$

We have introduced a cutoff in the bare functions to regularize any UV sub-divergences. The renormalized parameters $m, M$ and $g$ may or may not depend on $\kappa$ according to the specific renormalization procedure that we use. In the case at hand in four dimensions we may renormalize $m$ and $M$ on the mass shell without running into any problems in the limit $t \rightarrow \infty$. Similarly, as mentioned above no explicit $g_{B}$ renormalization is needed though one can certainly implement one if it turns out to be convenient. One may also introduce wavefunction renormalizations $Z_{\phi}(\kappa)$ and $Z_{\psi}(\kappa)$ for the fields $\phi$ and $\psi$ respectively, but once again in four dimensions such renormalizations are not necessary. Note that we have suppressed in the notation dependence in the $Z$ factors on parameters other than $\kappa$, the specific functional dependence depending on the actual specific renormalization scheme we choose.

The functions $B_{t}^{i j k l}$ satisfy renormalization group equations

$$
\kappa \frac{d B_{t}^{i j k l}(s, t, \kappa)}{d \kappa}=\sum_{m, n} \gamma_{b, t}^{i j m n}(\kappa) B_{t}^{m n k l}(s, t, \kappa),
$$

where $\gamma_{b, t}(\kappa)=\left(d Z_{b, t}(\kappa) / d \ln \kappa\right) \cdot Z_{b, t}^{-1}$ summarizes the anomalous scaling behaviour of the functions with respect to $\kappa$.

To produce explicit results we introduce a specific renormalization procedure to fix the values of the renormalization constants. We choose

$$
\begin{gathered}
\Gamma^{\psi \psi}\left(p^{2}=-M^{2}\right)=0 \\
\Gamma^{\phi \phi}\left(p^{2}=-m^{2}\right)=0 \\
\left(\begin{array}{cc}
B_{t}^{\phi \phi \phi \phi}(s, t=\kappa, \kappa) & B_{t}^{\phi \phi \psi \psi}(s, t=\kappa, \kappa) \\
B_{t}^{\psi \psi \psi \phi \phi}(s, t=\kappa, \kappa) & B_{t}^{\psi \psi \psi \psi \psi}(s, t=\kappa, \kappa)
\end{array}\right)=\left(\begin{array}{cc}
\frac{g_{B}^{2}}{\kappa+M^{2}} & \frac{g_{B}^{2}}{\kappa+m^{2}} \\
\frac{g_{B}^{2}}{\kappa+m^{2}} & 0
\end{array}\right)
\end{gathered}
$$

The remaining effective interactions of the type $B_{t}^{\phi \psi \phi \psi}$ etc. do not mix with the above and will not be considered in the following. Note that the normalization conditions (10-12) are explicitly $s$ and $t($ via $\kappa$ ) dependent. In this sense they are an environmentally friendly set of normalization conditions. The motivation behind them is that we are seeking a RG map to a region of parameter space where a perturbative calculation is possible. The "mean field" regime will always offer such a region if the map to it can be perturbatively constructed. That this map can be used to give perturbative control over the Green's functions for arbitrary values of $t$ will of course put a constraint on the value of the arbitrary renormalization scale $\kappa$ we choose. 
With the normalization conditions (12) in the large $t$ limit one finds to one loop

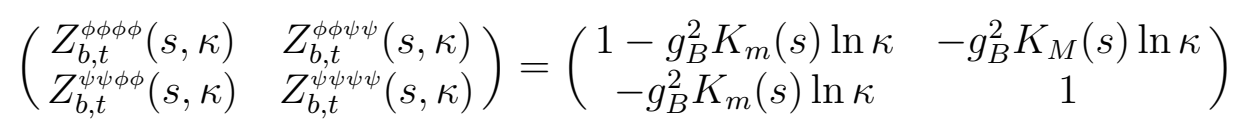

where the functions $K_{m}$ and $K_{M}$ are given by two-dimensional one loop diagrams:

$$
K_{\mu}(s)=\frac{1}{16 \pi^{2}} \int_{0}^{1} \frac{d \beta}{\beta(1-\beta) s+\mu^{2}} .
$$

The anomalous dimension matrix is

$$
\gamma_{b, t}=\left(\begin{array}{cc}
-g_{B}^{2} K_{m}(s) & -g_{B}^{2} K_{M}(s) \\
-g_{B}^{2} K_{m}(s) & 0
\end{array}\right)
$$

which leads to the following flow equations

$$
\begin{aligned}
& \left(\begin{array}{ll}
d B_{t}^{\phi \phi \phi \phi}(s, t, \kappa) / d \ln \kappa & d B_{t}^{\phi \phi \psi \psi \psi}(s, t, \kappa) / d \ln \kappa \\
d B_{t}^{\psi \psi \phi \phi \phi}(s, t, \kappa) / d \ln \kappa & d B_{t}^{\psi \psi \psi \psi}(s, t, \kappa) / d \ln \kappa
\end{array}\right)
\end{aligned}
$$

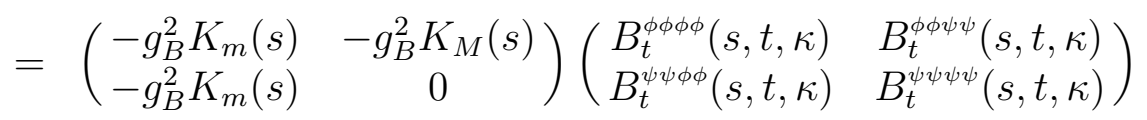

It is straightforward to solve these equations using as initial conditions the normalization conditions (12). One finds

$$
\begin{aligned}
& B_{t}^{\phi \phi \phi \phi}(s, t, \kappa) \\
& =\frac{g_{B}^{2}}{2 \kappa}\left(1+\frac{\left(1+2 \frac{K_{M}(s)}{K_{m}(s)}\right)}{\left(1+4 \frac{K_{M}(s)}{K_{m}(s)}\right)^{\frac{1}{2}}}\right)\left(\frac{t}{\kappa}\right)^{\alpha_{1}(s)}+\frac{g_{B}^{2}}{2 \kappa}\left(1-\frac{\left(1+2 \frac{K_{M}(s)}{K_{m}(s)}\right)}{\left(1+4 \frac{K_{M}(s)}{K_{m}(s)}\right)^{\frac{1}{2}}}\right)\left(\frac{t}{\kappa}\right)^{\alpha_{2}(s)}, \\
& B_{t}^{\psi \psi \psi \phi \phi}(s, t, \kappa)=B_{t}^{\phi \phi \psi \psi \psi}(s, t, \kappa) \\
& =\frac{g_{B}^{2}}{2 \kappa}\left(1+\frac{1}{\left(1+4 \frac{K_{M}(s)}{K_{m}(s)}\right)^{\frac{1}{2}}}\right)\left(\frac{t}{\kappa}\right)^{\alpha_{1}(s)}+\frac{g_{B}^{2}}{2 \kappa}\left(1-\frac{1}{\left(1+4 \frac{K_{M}(s)}{K_{m}(s)}\right)^{\frac{1}{2}}}\right)\left(\frac{t}{\kappa}\right)^{\alpha_{2}(s)}, \\
& B_{t}^{\psi \psi \psi \psi}(s, t, \kappa)=\frac{g_{B}^{2}}{\kappa\left(1+4 \frac{K_{M}(s)}{K_{m}(s)}\right)^{\frac{1}{2}}}\left(\frac{t}{\kappa}\right)^{\alpha_{1}(s)}-\frac{g_{B}^{2}}{\kappa\left(1+4 \frac{K_{M}(s)}{K_{m}(s)}\right)^{\frac{1}{2}}}\left(\frac{t}{\kappa}\right)^{\alpha_{2}(s)},
\end{aligned}
$$

where the Regge trajectories $\alpha_{1}$ and $\alpha_{2}$ are

$$
\begin{aligned}
& \alpha_{1}=\frac{g_{B}^{2} K_{m}(s)}{2}\left(1+\left(1+4 \frac{K_{M}(s)}{K_{m}(s)}\right)^{\frac{1}{2}}\right)-1, \\
& \alpha_{2}=\frac{g_{B}^{2} K_{m}(s)}{2}\left(1-\left(1+4 \frac{K_{M}(s)}{K_{m}(s)}\right)^{\frac{1}{2}}\right)-1 .
\end{aligned}
$$


It can be shown that these solutions correspond to the summation of leading logs. An entirely analogous consideration yields the functions $B_{u}^{i j k l}(s, u, \kappa)$ which turn out to be the same as the above with the simple change $t \rightarrow-t$. The functions $B_{B, s}^{i j k l}(t, u)$ on the other hand do not need renormalization as their crossover is completely controllable within perturbation theory.

Finally then we find the two-particle-two-particle $S$-matrix elements at one loop in the large $t$ limit to be

$$
\begin{aligned}
& \tilde{\Gamma}^{\phi \phi \phi \phi}(s, t, \kappa)=\frac{g_{B}^{2}}{s+M^{2}}+\frac{g_{B}^{2}}{2 \kappa}\left(1+\frac{\left(1+2 \frac{K_{M}(s)}{K_{m}(s)}\right)}{\left(1+4 \frac{K_{M}(s)}{K_{m}(s)}\right)^{\frac{1}{2}}}\right)\left\{\left(\frac{t}{\kappa}\right)^{\alpha_{1}(s)}+\left(-\frac{t}{\kappa}\right)^{\alpha_{1}(s)}\right\} \\
& +\frac{g_{B}^{2}}{2 \kappa}\left(1-\frac{\left(1+2 \frac{K_{M}(s)}{K_{m}(s)}\right)}{\left(1+4 \frac{K_{M}(s)}{K_{m}(s)}\right)^{\frac{1}{2}}}\right)\left\{\left(\frac{t}{\kappa}\right)^{\alpha_{2}(s)}+\left(-\frac{t}{\kappa}\right)^{\alpha_{2}(s)}\right\}, \\
& \tilde{\Gamma}^{\phi \phi \psi \psi}(s, t, \kappa)=\tilde{\Gamma}^{\psi \psi \phi \phi}(s, t, \kappa)=\frac{g_{B}^{2}}{2 \kappa}\left(1+\frac{1}{\left(1+4 \frac{K_{M}(s)}{K_{m}(s)}\right)^{\frac{1}{2}}}\right)\left\{\left(\frac{t}{\kappa}\right)^{\alpha_{1}(s)}+\left(-\frac{t}{\kappa}\right)^{\alpha_{1}(s)}\right\} \\
& +\frac{g_{B}^{2}}{2 \kappa}\left(1-\frac{1}{\left(1+4 \frac{K_{M}(s)}{K_{m}(s)}\right)^{\frac{1}{2}}}\right)\left\{\left(\frac{t}{\kappa}\right)^{\alpha_{2}(s)}+\left(-\frac{t}{\kappa}\right)^{\alpha_{2}(s)}\right\} \text {, } \\
& \tilde{\Gamma}^{\psi \psi \psi \psi}(s, t, \kappa)=\frac{g_{B}^{2}}{\kappa\left(1+4 \frac{K_{M}(s)}{K_{m}(s)}\right)^{\frac{1}{2}}}\left\{\left(\frac{t}{\kappa}\right)^{\alpha_{1}(s)}+\left(-\frac{t}{\kappa}\right)^{\alpha_{1}(s)}\right\} \\
& -\frac{g_{B}^{2}}{\kappa\left(1+4 \frac{K_{M}(s)}{K_{m}(s)}\right)^{\frac{1}{2}}}\left\{\left(\frac{t}{\kappa}\right)^{\alpha_{2}(s)}+\left(-\frac{t}{\kappa}\right)^{\alpha_{2}(s)}\right\} .
\end{aligned}
$$

Before we make our conclusions we will present the full crossover function $B_{t}^{\phi \phi \phi \phi}(s, t, \kappa)$ at one loop. For the sake of simplicity, we consider here a theory $\left(g_{B} / 3 !\right) \phi^{3}$, leaving a fuller discussion and extension to other cubic theories for another publication. As mentioned above, apart from a renormalization to get rid of the mass divergence, there is no need to have a $t$ dependent renormalization of the coupling or the mass. However, in order to arrive at a compact form for the crossover function here we will implement the normalization conditions

$$
\begin{gathered}
\Gamma^{\phi \phi}\left(p^{2}=\kappa, \kappa\right)=\kappa+m^{2}(\kappa), \\
\Gamma^{\phi \phi \phi}(t=\kappa, \kappa)=g(\kappa), \\
B_{t}^{\phi \phi \phi \phi}(s, t=\kappa, \kappa)=\frac{g^{2}(\kappa)}{\kappa+m^{2}(\kappa)} .
\end{gathered}
$$

One finds for $B_{t}^{\phi \phi \phi \phi}$

$$
B_{t}^{\phi \phi \phi \phi}(s, t, \kappa)=\frac{g^{2}(t)}{t+m^{2}(t)} \exp \int_{\kappa}^{t} \frac{d \kappa^{\prime}}{\kappa^{\prime}} g^{2}\left(\kappa^{\prime}\right) f_{1}\left(\kappa^{\prime}\right)
$$


where

$$
\begin{gathered}
g^{2}(t)=\frac{g^{2}(\kappa)}{1-2 g^{2}(\kappa)\left(f_{2}(t)-f_{2}(\kappa)\right)}, \\
m^{2}(t)=m^{2}(\kappa)+\int_{\kappa}^{t} \frac{d \kappa^{\prime}}{\kappa^{\prime}} g^{2}\left(\kappa^{\prime}\right) f_{3}\left(\kappa^{\prime}\right) .
\end{gathered}
$$

The function $f_{2}$ is just the four dimensional one loop coupling correction, whilst the functions $f_{1}$ and $f_{3}$ are given by

$$
f_{1}(\kappa)=\kappa \frac{\partial}{\partial \kappa}\left(\left(\kappa+m^{2}(\kappa)\right) h_{1}(\kappa)\right), \quad f_{3}(\kappa)=\kappa \frac{\partial}{\partial \kappa} h_{3}(\kappa)
$$

$h_{1}$ being the one loop box diagram, and $h_{3}$ the one loop mass correction. The function (28) crosses over to the expression corresponding to (17) in the asymptotic limit $t \gg \kappa \gg m^{2}$. In the small $t$ limit, if $g(\kappa) \ll 1$, then (28) just yields the perturbative one loop expression for $B_{t}^{\phi \phi \phi \phi}$ for the theory renormalized according to the above normalization conditions.

To summarize, we have presented in this letter an environmentally friendly renormalization that is capable of capturing the crossover between large and small $t$ in the two-particletwo-particle scattering amplitudes. We derived in particular the asymptotic behaviour in the case of a theory $\phi^{2} \psi$ with unequal masses, including the relevant Regge trajectories and derived a crossover scaling function for a $\phi^{3}$ theory. We hope that it is clear that the methodology is capable of producing a wide variety of results for the kinematical dimensional reduction that occurs in the Regge limit. In future publications we will present applications to more realistic theories and also implement an RG that is capable of accessing dual amplitudes.

\section{Acknowledgements}

A.W. would like to thank the Instituto de Ciencias Nucleares for kind hospitality.

\section{References}

[1] E.A. Kuraev, L.N. Lipatov and V.S. Fadin, Sov. Phys. JETP 45 (1977) 199.

[2] "The Analytic S-matrix", R.J. Eden, P.V. Landshoff, D.I. Olive and J.C. Polkinghorne; (CUP 1966).

[3] A.A. Migdal, A.M. Polyakov and K.A. Ter-Martirosyan, Phys. Lett. B48 (1974) 239.

[4] D. O'Connor and C.R. Stephens, Nucl. Phys. B360 (1991) 297; Int. J. Mod. Phys. A9 (1994) 2805; Phys. Rev. Lett. 72 (1994) 506.

[5] D. O'Connor, C.R. Stephens and F. Freire, Mod. Phys. Lett. A8 (1993) 1779;

M.A. van Eijck, C.R. Stephens and C.G. van Weert, Mod. Phys. Lett. A9 (1994) 309. 


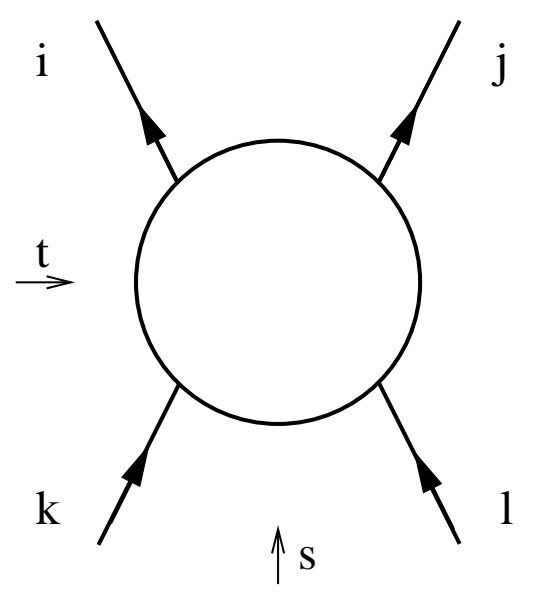

Figure 1 